This is the post-print version of the article published as:

Nat. Mater., 2016, DOI: 10.1038/nmat4652

http://www.nature.com/nmat/journal/v15/n9/abs/nmat4652.html

\title{
Tuning the energetics and tailoring optical properties of silver clusters confined in zeolites
}

\author{
Oliver Fenwick ${ }^{1 \S \dagger}$, Eduardo Coutiño-Gonzalez ${ }^{2 \dagger}$, Didier Grandjean ${ }^{3}$, Wouter Baekelant ${ }^{2}$, \\ Fanny Richard ${ }^{1}$, Sara Bonacchi ${ }^{1}$, Dirk De Vos ${ }^{4}$, Peter Lievens ${ }^{3}$, Maarten Roeffaers ${ }^{4}$, Johan \\ Hofkens $^{2 *}$, Paolo Samori ${ }^{1 *}$ \\ ${ }^{1}$ ISIS \& icFRC, Université de Strasbourg \& CNRS, 8, allée Gaspard Monge, 67000 Strasbourg, \\ France.E-mail: samori@unistra.fr \\ ${ }^{2}$ Department of Chemistry, KU Leuven, Celestijnenlaan 200F, B-3001 Leuven, Belgium. E- \\ mail: Johan.Hofkens@chem.kuleuven.be \\ ${ }^{3}$ Laboratory of Solid State Physics and Magnetism, KU Leuven, Celestijnenlaan 200D, B-3001 \\ Leuven, Belgium \\ ${ }^{4}$ Department of of Microbial and Molecular Systems, KU Leuven, Celestijnenlaan 200F, B- \\ 3001 Leuven, Belgium. E-mail: Maarten.Roeffaers@biw.kuleuven.be \\ ${ }^{\S}$ Current address: School of Engineering and Materials Science, Queen Mary University of \\ London, Mile End Road, London E1 4NS, United Kingdom. \\ ${ }^{\dagger}$ These authors contributed equally to the work.
}

The integration of metal atoms and clusters in well-defined dielectric cavities is a powerful strategy to impart novel properties to them that depend on the size and geometry of the confined space as well as on metal-host electrostatic interactions. Here, we unravel the dependence of the electronic properties of metal clusters on space confinement by studying the ionisation potential of silver clusters embedded in four different zeolite environments over a range of silver concentrations. Extensive characterisation reveals a strong influence of silver loading and host environment on the cluster ionisation potential, which is also correlated to the cluster's optical and structural properties. Through fine-tuning of the zeolite host environment, we demonstrate photoluminescence quantum yields approaching 
unity. This work extends our understanding of structure-property relationships of small metal clusters and applies this understanding to develop highly photoluminescent materials with potential applications in optoelectronics and bioimaging.

\section{MAIN TEXT}

Self-assembly is a well-established approach to build up 1, 2 or 3 dimensional hierarchical structures with controlled organisation at distinct length scales. It is therefore an ideal method to guide both the chemical and physical properties of a system, surpassing the scope of properties that can be obtained in conventional liquid or solid-state environments. A particularly interesting method to direct the self-assembly process is the use of confined spaces such as pores, channels or cages which impose restrictions on the final geometry and dimensions of an embedded functional self-assembled system.

Small noble metal clusters can exhibit molecule-like behaviour in terms of their electronic transitions, and certain clusters of silver atoms have proved particularly interesting due to their pronounced catalytic ${ }^{1}$ and optical properties. ${ }^{2}$ However, difficulty in obtaining monodisperse cluster sizes and their tendency to aggregate makes harnessing these exciting properties for applications challenging. To circumvent this problem, a number of stabilising strategies have been proposed including soft matter approaches based on DNA, ${ }^{2,3}$ polyphosphates, ${ }^{4,5}$ organic polymers ${ }^{6}$ and peptides, ${ }^{7}$ and more rigid approaches relying on glasses ${ }^{8,9}$ and zeolites. ${ }^{10-13}$ Zeolites are readily available naturally occurring minerals, but can also be synthesised in industrial quantities at low cost with a tailored structure. They are attractive as hosts for silver clusters because of the relative ease with which $\mathrm{Ag}^{+}$ions can be incorporated by means of ion exchange, as well as their well-defined crystal structures with 
cages and channels of molecular dimensions. The tunable combination of topology, framework charge, the coordinating properties of the framework oxygen atoms and the extra-framework counter-cations all work to stabilise clusters of certain sizes and geometries.

In advancing the state-of-the-art for Ag cluster-containing zeolites for fluorescence applications it is mandatory to master the versatility of their chemistry to fully exploit the properties that emerge due to space confinement. Autoreduction processes during calcination of zeolites have been identified in silver cluster formation, with the required electrons originating from the expulsion of oxygen atoms from the zeolite framework or from the oxidation of hydration water to oxygen. ${ }^{14}$ However, despite much effort, relatively little is known about how to control the nuclearity and location of the clusters within zeolites. Moreover, the relationship between the electronic and structural properties of such minuscule metal structures has not been fully understood. In this paper we systematically study the effect of space confinement on Ag clusters formed inside zeolites. In particular, we explore the role of framework topology, degree of silver exchange, negative framework charge, and the cationic species in the parent zeolite. With control over these four key parameters we correlate the optical properties with the ionisation potentials of the silver clusters, and ultimately tune the emission from the green to deep red, demonstrating photoluminescence quantum yields (PLQYs) of close to $100 \%$.

\section{Preparation of silver cluster-containing zeolites}

We focussed our attention on two prototypical framework topologies, Linde type A (LTA) and faujasite (FAU) (Figure 1a). Both contain sodalite cages, but differ in the secondary building units interconnecting these cages. $\mathrm{Ag}_{2}{ }^{\mathrm{n}}, \mathrm{Ag}_{3}{ }^{\mathrm{n}}, \mathrm{Ag}_{4}{ }^{\mathrm{n+}}$ and $\mathrm{Ag}_{8}{ }^{\mathrm{n}+}$ clusters have all 
been reported in FAU frameworks, ${ }^{15-21}$ whilst $\mathrm{Ag}_{3}{ }^{\mathrm{n}+}$ and $\mathrm{Ag}_{6}{ }^{\mathrm{n+}}$ clusters are frequently identified in LTA frameworks. ${ }^{19,22}$ The two faujasite frameworks chosen for this study, FAUX and FAUY, differ in Si/Al ratio of the framework (1.2 for FAUX and 2.7 for FAUY from data provided by the suppliers) enabling us to explore the role of framework charge. The two LTA zeolites, $3 \mathrm{~A}$ and $4 \mathrm{~A}$, differ in the alkali metal charge-balancing cation $\left(\mathrm{Na}^{+}\right.$and $\mathrm{K}^{+}$ respectively).

Starting from the parent zeolites, silver exchange was carried out in an aqueous silver nitrate solution before calcination at $450^{\circ} \mathrm{C}$ in air to allow cluster formation. We obtained fully- and partially-exchanged zeolites which we refer to according to their nominal silver content relative to the dehydrated unit cells normalised to $24 \mathrm{~T}$-atoms (the tetrahedrally coordinated $\mathrm{Si}$ and $\mathrm{Al}$ atoms). These are: $\left(\mathrm{Ag}^{+}{ }_{x} \mathrm{Na}^{+}{ }_{11-\mathrm{x}}\right)\left[\mathrm{Al}_{11} \mathrm{Si}_{13} \mathrm{O}_{48}\right]$, referred to as FAUX[Ag $]$; $\left(\mathrm{Ag}^{+}{ }_{x} \mathrm{Na}^{+}{ }_{6.5-\mathrm{x}}\right)\left[\mathrm{Al}_{6.5} \mathrm{Si}_{17.5} \mathrm{O}_{48}\right]$, referred to as FAUY $\left[\mathrm{Ag}_{\mathrm{x}}\right] ;\left(\mathrm{Ag}^{+}{ }_{\mathrm{x}} \mathrm{K}^{+}{ }_{12-\mathrm{x}}\right)\left[\mathrm{Al}_{12} \mathrm{Si}_{12} \mathrm{O}_{48}\right]$, referred to as $3 \mathrm{~A}\left[\mathrm{Ag}_{\mathrm{x}}\right]$; and $\left(\mathrm{Ag}^{+}{ }_{x} \mathrm{Na}^{+}{ }_{12-\mathrm{x}}\right)\left[\mathrm{Al}_{12} \mathrm{Si}_{12} \mathrm{O}_{48}\right]$, referred to as $4 \mathrm{~A}\left[\mathrm{Ag}_{\mathrm{x}}\right]$. For simplicity, the nominal degree of exchange used in our nomenclature assumes complete exchange of the cations in the exchange solution.

The composition of the calcined zeolites was examined by XPS noting that the ratio of silver to alkali metal at intermediate exchange levels differs slightly from the nominal values (Supplementary Figures 1 and 2). Also noteworthy is that sodium was detected in the $3 \mathrm{~A}\left[\mathrm{Ag}_{0}\right]$ unexchanged zeolite provided by the supplier (i.e. alkali metal composition is $15 \%$ $\mathrm{Na}$ and $85 \% \mathrm{~K}$ as a proportion of the ion exchange capacity, Supplementary Figure 3). As a first characterisation of the bonding environment of silver, we followed the Auger spectra of the calcined zeolite. Auger electrons are emitted in a three electron process, making them highly sensitive to the chemical bonding environment. Modified Auger parameters (MAPs) 
(equal to the sum of the binding energy of $3 d_{5 / 2}$ electrons and the kinetic energy of $\mathrm{M}_{4} \mathrm{~N}_{4,5} \mathrm{~N}_{4,5}$ Auger electrons) quantify the shift of atomic orbitals due to their chemical environment without susceptibility to charging effects. ${ }^{21}$ The MAPs of silver in the zeolites are $721.9 \pm 0.4 \mathrm{eV}$ for $3 \mathrm{~A}\left[\mathrm{Ag}_{\mathrm{x}}\right], 722.2 \pm 0.4 \mathrm{eV}$ for FAUX[Ag $]$, and $723.3 \pm 0.4 \mathrm{eV}$ for FAUY $\left[\mathrm{Ag}_{\mathrm{x}}\right]$ (Supplementary Table 1) which represent a large chemical shift ( 3 - $5 \mathrm{eV}$ ) compared to silver metal $(\mathrm{MAP}=726.0 \mathrm{eV})$ and a $1-2 \mathrm{eV}$ chemical shift compared to silver (I) oxide (MAP $=724.1 \mathrm{eV})$. These chemical shifts are indicative of small Ag clusters. ${ }^{20}$ Larger silver nanoparticles have been detected by XPS in other zeolite frameworks, ${ }^{23}$ but in our zeolites we can rule out even small quantities of silver nanoparticles from the large chemical shifts and the absence of metallic features in the Auger spectrum (Figure $1 \mathrm{~b}$ and Supplementary Figure 4).

\section{Tuning electro-optical properties}

We used photoelectron spectroscopy in air (PESA) to quantify the ionisation potentials (IPs) of metal clusters in well-defined confined spaces. Our results provide unambiguous evidence of a pronounced silver loading effect (Figure 2a), with IPs gradually increasing with silver content by up to $0.5 \mathrm{eV}$, as well as evidence of an influence of the zeolitic host. In determining the origin of the silver loading effect, one must consider that silver-exchanged zeolites are bimetallic systems with both silver and alkali metal cations/clusters. The decrease of the IP with decreasing silver loading is most likely due to the reduced photoelectron signal coming from silver clusters combined with an increased signal from alkali metal $(\mathrm{Na}, \mathrm{K})$ ions (or clusters ${ }^{24,25}$, Supplementary Figures 5-8). This shifts the measured IP towards the value of the latter, noting that the IPs of sodium and potassium clusters $^{26-29}$ and metals ${ }^{30}$ are lower than those of silver. 
In order to study the effect of the framework type, we focussed our attention on fully exchanged zeolites where silver is the only extra-framework metal. It is clear that the IPs of clusters in the four zeolites are markedly different, with clusters in FAU frameworks having larger IPs than LTA (FAUY $\left[\mathrm{Ag}_{6.5}\right]$ the largest being $5.45 \mathrm{eV}$ and $3 \mathrm{~A}\left[\mathrm{Ag}_{12}\right]$ the smallest being $5.16 \mathrm{eV}$, Figure $2 \mathrm{~b})$. These results clearly demonstrate that self-assembly of silver clusters in the confined space of zeolites is strongly affected both by the geometry and the charge density of the environment.

Excitation-emission spectra of the LTA zeolites (Figure 3a) show the emergence of new bathochromically shifted peaks at progressively higher silver loading suggesting the formation of larger clusters. On the other hand, FAU zeolites show a remarkable resilience to larger cluster formation at higher silver loadings as ascertained from the lack of emergence of new emission peaks (Figure 3b, Supplementary Figures 9 and 10). It is therefore reasonable that the IPs of clusters in fully-loaded $3 \mathrm{~A}$ and $4 \mathrm{~A}$ zeolites are lower than those in FAUX and FAUY since the HOMO levels of silver clusters are known to increase with nuclearity. ${ }^{31}$ To further support this, we show the electron spin resonance (ESR)

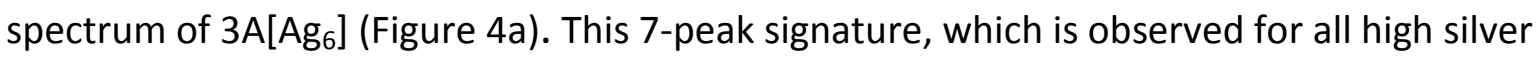
content zeolites $3 \mathrm{~A}\left[\mathrm{Ag}_{6-12}\right]$ shows the coupling of the electron spin with six equivalent nuclear spins. Such signals have been associated with the presence of $\mathrm{Ag}_{6}{ }^{+}$clusters. ${ }^{14,22}$ Low silver content zeolites $3 \mathrm{~A}\left[\mathrm{Ag}_{<6}\right]$ are ESR silent (Figure $4 \mathrm{~b}$ ) indicating diamagnetic clusters widely reported to be $\mathrm{Ag}_{3}{ }^{+19} \cdot{ }^{19}$ This is in line with our previous observations in silverexchanged zeolite $4 A .{ }^{14}$ Our extended X-ray absorption fine structure (EXAFS) findings (Figure 4c, d vide infra) confirm ESR silent $\mathrm{Ag}_{4}{ }^{\mathrm{n}+}$ clusters in FAU zeolites across all silver loadings (Supplementary Figure 11). Furthermore, analysis of the relationship between the 
IPs of the fully loaded zeolites and the peak emission energy of the clusters (Figure 2b), reveals that an increase in the IP is accompanied by a corresponding increase in the emission energy. In particular, a straight-line fit to this data yields a gradient close to two $(1.8 \pm 0.3)$, implying that about half of the framework-induced change in emission energy is due to a shift in the HOMO level of the luminescent clusters and about half is due to a shift of their LUMO in the opposite direction.

The optical properties of the fully exchanged zeolites $3 A\left[\mathrm{Ag}_{12}\right]$ and $4 \mathrm{~A}\left[\mathrm{Ag}_{12}\right]$ unexpectedly differ (Figure 3a,b) despite nominally identical compositions. The origin of this anomaly appears to be residual quantities of $\mathrm{Na}^{+}$and $\mathrm{K}^{+}$in $3 \mathrm{~A}\left[\mathrm{Ag}_{12}\right]$ amounting to $15 \%$ of the total metal ions present (Supplementary Figure 3). To test this, we carried out ion exchange in an excess of silver nitrate. XPS analysis confirms $>99 \%$ silver exchange. The resulting photoluminescence properties of $3 \mathrm{~A}\left[\mathrm{Ag}_{\text {excess }}\right]$ and $4 \mathrm{~A}[\mathrm{Ag}$ excess $]$ (Figure 5) are strikingly similar with a single strong emission in the red, confirming the important role played by even small amounts (intentional or otherwise) of other counter-cations in silver cluster formation.

Our previous studies revealed that higher photoluminescence quantum yields (PLQYs) can be obtained by confining Ag clusters inside the FAU topology rather than LTA. In this study, we find that the PLQY of FAUX and FAUY zeolites are similar at higher silver loadings (40$60 \%$ for $\left[A g_{x}\right] x \geq 3$ ), but deviate significantly at lower loadings (Figure $3 c$ ). For FAUX, the PLQY increases from $1 \%$ at $x=1$ before reaching a plateau of about $50 \%$ at $x \approx 5$, whilst for FAUY the PLQY at the lowest loading $(x=0.5)$ is $97.4 \pm 2.1 \%$ and decreases to a plateau at higher silver concentrations. The PLQY in FAUY $\left[\mathrm{Ag}_{0.5}\right]$ is by far the highest quantum yield observed for any silver species contained in zeolite frameworks ${ }^{10}$ and also exceeds the best quantum yields of silver clusters stabilised in other materials. ${ }^{2,3,32,33}$ Consequently, this 
result is an important milestone in the development of these materials as secondary emitters for lighting and for bio-imaging, ${ }^{33,34}$ and brings the performance of metal clustercontaining zeolites to the level of certain dye-containing zeolites. ${ }^{35}$ Furthermore, achieving these high efficiencies at low silver concentrations inside the rigid zeolite framework makes them cost-effective and also reduces potential toxicity risks.

\section{Physical structure of the silver clusters}

To understand more about the origin of these exceptional PLQYs in FAU zeolites, we characterized the clusters using EXAFS. EXAFS analysis is a powerful method for obtaining atomic-scale information on clusters and their environment, including metal-metal and metal-ligand bonding, and estimation of cluster size. ${ }^{36}$ We found that a model based on a three-shell structure $(\mathrm{Ag}-\mathrm{O}, \mathrm{Ag}-\mathrm{Ag} \text {, and } \mathrm{Ag}-\mathrm{Al} / \mathrm{Si})^{37}$ displays the best agreement with the results obtained from our EXAFS analysis. Further details including curve-fitting parameters are given in Supplementary Section 5.

In the FAU topology extra-framework cations are mainly located in a few well-defined sites conventionally designated as site I (the centre of the hexagonal prism), site II (coordinated to a single six-ring -S6R- in the large supercage) and the sites I' and II', opposite the sites I and II, but inside the sodalite cage (Supplementary Figure 12). ${ }^{18,38,39}$ Having first fitted the data in both $\mathrm{k}^{2}$ and $\mathrm{k}^{3}$ spaces, we found that $\mathrm{k}^{3}$ weighting gave the most useful information on silver clusters due to its sensitivity to heavy atoms. Figure $4 c, d$ show the $k^{3}$-weighted EXAFS signal $\left(k^{3} \chi(k)\right)$ and the corresponding phase-corrected Fourier transform (FT) best fits of heat-treated FAUY $\left[\mathrm{Ag}_{0.5}\right]$. The first peak in Figure $4 \mathrm{~d}$ corresponds to multiple oxygen contributions reflecting the coordination of silver atoms in positions II, II' $\left(\mathrm{Ag}_{R}\right)$ and I $\left(\mathrm{Ag}_{\mathrm{P}}\right)$ 
with framework oxygen atoms as well as the coordination of silver forming the clusters $\left(\mathrm{Ag}_{\mathrm{C}}\right)$ with extra-framework water molecules (Supplementary Table 2).

The second peak in the FT is mostly composed of two Ag-Si/Al contributions corresponding to $\mathrm{Ag}_{\mathrm{R}}$ and $\mathrm{Ag}$ p silver ions. ${ }^{17}$ In addition to the large $\mathrm{Al} / \mathrm{Si}$ contribution, a weaker $\mathrm{Ag}-\mathrm{Ag}$ at a distance of $2.95 \AA$ can also be detected in the second FT multipeak. This contribution corresponds to the remaining silver atoms $\left(\mathrm{Ag}_{\mathrm{C}}\right)$ forming clusters located inside the sodalite cage. The $\mathrm{Ag}$ coordination around $\mathrm{Ag}_{\mathrm{C}}$ atoms is 2.84 suggesting that the cluster nuclearity is very close to 4. Two long distance Ag shells were added to our model corresponding to distances from $\mathrm{Ag}_{C}$ atoms to $\mathrm{Ag}_{R}$ and $\mathrm{Ag}_{\mathrm{P}}$ cations. No Na shell could be clearly detected in the EXAFS model.

It emerges from our data that, in FAUY[Ag0.5], ca. $67 \%$ of silver atoms $\left(\mathrm{Ag}_{\mathrm{c}}\right)$ form $\mathrm{Ag}_{4}$ clusters inside the sodalite cages in which each Ag atom is bonded to 2.2 water molecules with the remaining ca. $33 \%$ of silver atoms being non-cluster-forming $\mathrm{Ag}_{\mathrm{R}}$ (ca. $18 \%$ ) and $\mathrm{Ag}_{\mathrm{P}}$ (ca. $15 \%$ ) cations.

Similar EXAFS analysis of the fully Ag-exchanged sample $\mathrm{FAUY}\left[\mathrm{Ag}_{6.5}\right]$ also reveals $\mathrm{Ag}_{4}$ cluster structure, but with slightly fewer silver atoms involved in cluster formation (ca. 62\%). On the other hand, a significantly larger Debye-Waller factor $\left(A=2 \sigma^{2}\right)$ associated with $\mathrm{Ag}_{\mathrm{c}}-\mathrm{Ag}_{\mathrm{c}}$ shells is observed in FAUY $\left[\mathrm{Ag}_{6.5}\right]\left(0.044 \AA^{2}\right)$ than in FAUY $\left[\mathrm{Ag}_{0.5}\right]\left(0.026 \AA^{2}\right)$. The Debye-Waller factor describes the attenuation of X-rays by thermal motion and by static displacements of the atoms. This indicates that $\mathrm{Ag}_{4}$ clusters are significantly more ordered in $\mathrm{FAUY}\left[\mathrm{Ag}_{0.5}\right]$ than in fully-loaded FAUY[Ag 6.5$]$. Taken together, these observations strongly suggest that the increase in quantum efficiency of Ag-FAUY zeolites with decreasing silver loading is driven by increasing order within the clusters rather than by changes in nuclearity of clusters or the 
proportion of silver atoms involved in cluster formation (though the latter has a small effect).

It is interesting to consider why the trend in PLQY for FAUX is so different. The absence of new peaks in the excitation-emission spectra with increasing silver loading indicates that the nuclearity of luminescent cluster formed in FAUX zeolites is not dependent on silver loading; a similar observation can be made for FAUY. We therefore consider that luminescent cluster formation must be hindered at low silver loading in FAUX. This is supported by EXAFS analysis (Supplementary Table 2, Supplementary Figure 12) which shows that Ag clusters have a nuclearity of about 4 in all our FAUX zeolites, but a significantly lower proportion of silver atoms form clusters at low silver loadings than at high loadings (ca. $57 \%$ in FAUX[Ag $\left.{ }_{1}\right]$ compared to ca. $73 \%$ in FAUX[Ag $\left.\left.{ }_{3}\right]\right)$. This is the opposite trend to FAUY and consistent with the drop-off in PLQY at low silver loadings in FAUX, though not enough by itself to explain such low efficiencies. In fact, $\mathrm{Na}^{+}$ions are more mobile than $\mathrm{Ag}^{+}$ions in FAUX, but the opposite is true for FAUY. ${ }^{40-43}$ The reason for this counterintuitive trend in ionic conduction is the trade-off between the Coulombic framework-cation interaction, which decreases for larger ionic radii, and cation-cation repulsion which increases with ionic radius and is more prominent in FAUX zeolites than FAUY due to the higher cation density. ${ }^{43}$ Cation-cation repulsion should decrease silver ion mobility with increasing silver content, which may be responsible for the greater degree of disorder in clusters in FAUY at high silver loading along with static effects such as poorer charge balance in the zeolite. Closer analysis of the EXAFS signal of FAUX[Ag 1$]$ reveals a sodium shell around the silver clusters which could not be detected in FAUY. This strongly suggests that sodium ions, which are more concentrated and more mobile in FAUX compared to FAUY, are playing a key role. Due to their high 
mobility relative to $\mathrm{Ag}^{+}$in $\mathrm{FAUX}$, they are able to move to sites inside the sodalite cages, interfering with silver cluster formation and affecting the electronic/geometric structure of silver clusters that do form. EXAFS also reveals that the sodium reduces the amount of water coordinated to the cluster which may also reduce PLQY. This highlights another important aspect to the role played by counter-ions in luminescent cluster formation.

\section{Conclusions and Outlook}

In this work we have shown that the rigid frameworks of zeolites provide a surprisingly versatile host for the stabilisation of silver clusters and enable a high degree of control over their optoelectronic properties. In particular, the degree of silver loading in the framework and the framework type allow the possibility to tune the IP over a range of $>0.5 \mathrm{eV}$, which we can also correlate with the optical properties. We have shown that, with the use of zeolite hosts, PLQYs can be optimised to nearly $100 \%$ by careful tuning of the mobilities of non-framework metal cations. The high quantum efficiencies of our best materials originate from an improved degree of order within the clusters and exceed all previous reports on luminescent silver clusters. This opens up potential applications for these materials in optoelectronics such as luminescent tags ${ }^{34}$ or secondary emitters in fluorescent lamps. The compatibility with aqueous environments, possibility of two-photon excitation and potential for down-scaling to nano-sized zeolites also makes them suitable for bio-imaging applications. ${ }^{14,44}$ Looking beyond this work we consider this an interesting system for catalysis since it combines the exceptional catalytic properties of well-defined oligomeric metal clusters ${ }^{45}$ with the selectivity of the porous zeolite network. ${ }^{44}$ Furthermore, we envisage the extension of luminescent silver-zeolite systems to other topologies such as EMT and EMT-FAU intergrowths. ${ }^{46}$ This work establishes the use of rigid confined spaces 
with tunable topological and electrostatic properties as a powerful method to direct

self-assembly and achieve enhanced material properties not accessible by other means.

\section{REFERENCES}

1. Martens J. A., et al. NOx abatement in exhaust from lean-burn combustion engines by reduction of NO2 over silver-containing zeolite catalysts. Angew. Chem. Int. Ed. 37, 19011903 (1998).

2. Vosch T., et al. Strongly emissive individual DNA-encapsulated Ag nanoclusters as singlemolecule fluorophores. Proc. Natl. Acad. Sci. USA 104, 12616-12621 (2007).

3. Richards C. I., et al. Oligonucleotide-stabilized Ag nanocluster fluorophores. J. Am. Chem. Soc. 130, 5038-5039 (2008).

4. Henglein A. Small-particle research - Physicochemical properties of extremely small colloidal metal and semiconductor particles. Chem. Rev. 89, 1861-1873 (1989).

5. Mulvaney P. \& Henglein A. Long-lived nonmetallic silver clusters in aqueous solution - a pulse-radiolysis study of their formation. J. Phys. Chem. 94, 4182-4188 (1990).

6. Diez I., et al. Blue, green and red emissive silver nanoclusters formed in organic solvents. Nanoscale 4, 4434-4437 (2012).

7. Yu J., Patel S. A. \& Dickson R. M. In vitro and intracellular production of peptideencapsulated fluorescent silver nanoclusters. Angew. Chem. 119, 2074-2076 (2007).

8. Borsella E., et al. Synthesis of silver clusters in silica-based glasses for optoelectronics applications. J. Non-Cryst. Solids 245, 122-128 (1999).

9. Shestakov M. V., et al. Lead silicate glass $\mathrm{SiO}_{2}-\mathrm{PbF}_{2}$ doped with luminescent $\mathrm{Ag}$ nanoclusters of a fixed site. RSC Adv. 4, 20699-20703 (2014).

10. Coutino-Gonzalez E., et al. Determination and optimization of the luminescence external quantum efficiency of silver-clusters zeolite composites. J. Phys. Chem. C 117, 6998-7004 (2013).

11. Grobet P. J. \& Schoonheydt R. A. ESR on silver clusters in zeolite A. Surf. Sci. 156, 893-898 (1985). 
12. Seifert R., Kunzmann A. \& Calzaferri G. The yellow color of silver-containing zeolite A. Angew. Chem. Int. Ed. 37, 1521-1524 (1998).

13. Wasowicz T. \& Michalik J. Reactions of silver atoms and clusters in Ag-NaA zeolites. Radiat. Phys. Chem 37, 427-432 (1991).

14. De Cremer G., et al. Characterization of fluorescence in heat-treated silver-exchanged zeolites. J. Am. Chem. Soc. 131, 3049-3056 (2009).

15. De Cremer G., et al. In situ observation of the emission characteristics of zeolite-hosted silver species during heat treatment. ChemPhysChem 11, 1627-1631 (2010).

16. Gellens L. R., Mortier W. J., Lissillour R. \& Lebeuze A. Electronic-structure of the silver clusters in zeolites of type $A$ and the faujasite type by molecular-orbital calculations. J. Phys. Chem. 86, 2509-2516 (1982).

17. Gellens L. R., Mortier W. J., Schoonheydt R. A. \& Uytterhoeven J. B. The nature of the charged silver clusters in dehydrated zeolites of type A. J. Phys. Chem. 85, 2783-2788 (1981).

18. Gellens L. R., Mortier W. J. \& Uytterhoeven J. B. On the nature of the charged silver clusters in zeolites of type A, type $X$ and type Y. Zeolites 1, 11-18 (1981).

19. Sun T. \& Seff K. Silver clusters and chemistry in zeolites. Chem. Rev. 94, 857-870 (1994).

20. Fonseca A. M. \& Neves I. C. Study of silver species stabilized in different microporous zeolites. Microporous Mesoporous Mater. 181, 83-87 (2013).

21. Gaarenstroom S. W. \& Winograd N. Initial and final-state effects in ESCA spectra of Cadmium and silver oxides. J. Chem. Phys. 67, 3500-3506 (1977).

22. Mayoral A., Carey T., Anderson P. A., Lubk A. \& Diaz I. Atomic resolution analysis of silver ion-exchanged zeolite A. Angew. Chem. Int. Ed. 50, 11230-11233 (2011).

23. Anson A., Maham Y., Lin C. C. H., Kuznicki T. M. \& Kuznicki S. M. XPS characterization of silver exchanged ETS-10 and mordenite molecular sieves. J. Nanosci. Nanotechnol. 9, 3134-3137 (2009).

24. Blake N. \& Stucky G. Alkali-metal clusters as prototypes for electron solvation in zeolites. J. Incl. Phenom. Macrocycl. Chem. 21, 299-324 (1995).

25. Kasai P. H. Electron spin resonance studies of $\mathrm{Y}$ - and X-ray-irradiated zeolites. J. Chem. Phys. 43, 3322-3327 (1965). 
26. Foster P. J., Leckenby R. E. \& Robbins E. J. The ionization potentials of clustered alkali metal atoms. J. Phys. B 2, 478-483 (1969).

27. Honea E. C., Homer M. L., Persson J. L. \& Whetten R. L. Generation and photoionization of cold $\mathrm{Na}_{n}$ clusters - $\mathrm{n}$ to 200. Chem. Phys. Lett. 171, 147-154 (1990).

28. Onwuagba B. N. Ionization potentials in alkali-metal clusters. II Nuovo Cimento D 13, 415-421 (1991).

29. Martins J. L., Buttet J. \& Car R. Equilibrium geometries and electronic-structures of small sodium clusters. Phys. Rev. Lett. 53, 655-658 (1984).

30. Kaye G. W. C. \& Laby T. H. Tables of physical and chemical constants, 16th edn, 1995.

31. Jackschath C., Rabin I. \& Schulze W. Electron impact ionization of silver clusters $A_{n}, n \leqq 36 . Z$. Phys. D: At., Mol. Clusters 22, 517-520 (1992).

32. Kuznetsov A. S., Tikhomirov V. K., Shestakov M. V. \& Moshchalkov V. V. Ag nanocluster functionalized glasses for efficient photonic conversion in light sources, solar cells and flexible screen monitors. Nanoscale 5, 10065-10075 (2013).

33. Choi S., Dickson R. M. \& Yu J. H. Developing luminescent silver nanodots for biological applications. Chem. Soc. Rev. 41, 1867-1891 (2012).

34. De Cremer G., et al. Optical Encoding of Silver Zeolite Microcarriers. Adv. Mater. 22, 957-960 (2010).

35. Devaux A., et al. Self-absorption and luminescence quantum yields of dye-zeolite L composites. J. Phys. Chem. C 117, 23034-23047 (2013).

36. Neidig M. L., et al. Ag K-Edge EXAFS Analysis of DNA-Templated Fluorescent Silver Nanoclusters: Insight into the Structural Origins of Emission Tuning by DNA Sequence Variations. J. Am. Chem. Soc. 133, 11837-11839 (2011).

37. Yamamoto T., Takenaka S., Tanaka T. \& Baba T. Stability of silver cluster in zeolite A and Y catalysts. 14th International Conference on X-Ray Absorption Fine Structure 190, 012171 (2009).

38. Gellens L. R., Mortier W. J. \& Uytterhoeven J. B. Oxidation and reduction of silver in zeolite Y - A structural study. Zeolites 1, 85-90 (1981). 
39. Smith J. V. Molecular Sieve Zeolites-I, vol. 101. American Chemical Society, 1974.

40. Mortier W. J. \& Schoonheydt R. A. Surface and solid-state chemistry of zeolites. Prog. Solid State Chem. 16, 1-125 (1985).

41. Freeman D. C. \& Stamires D. N. Electrical conductivity of synthetic crystalline zeolites. J. Chem. Phys. 35, 799-806 (1961).

42. Simon U. \& Franke M. E. Electrical properties of nanoscaled host/guest compounds. Microporous Mesoporous Mater. 41, 1-36 (2000).

43. Kalogeras I. M. \& Vassilikou-Dova A. Electrical properties of zeolitic catalysts. Defect Diffus. Forum 164, 1-36 (1998).

44. Awala H., et al. Template-free nanosized faujasite-type zeolites. Nat. Mater. 14, 447-451 (2015).

45. Corma A., et al. Exceptional oxidation activity with size-controlled supported gold clusters of low atomicity. Nat. Chem. 5, 775-781 (2013).

46. Ng E.-P., Chateigner D., Bein T., Valtchev V. \& Mintova S. Capturing Ultrasmall EMT Zeolite from Template-Free Systems. Science 335, 70-73 (2012).

\section{METHODS}

\section{Synthesis of luminescent silver exchanged zeolites.}

Synthesis of the silver-exchanged zeolites was carried out starting from $500 \mathrm{mg}$ of the zeolite material ( $3 \mathrm{~A}$ zeolite with $\mathrm{Si} / \mathrm{Al}=1 ; 4 \mathrm{~A}$ zeolite with $\mathrm{Si} / \mathrm{Al}=1$; $\mathrm{FAUX}$ zeolite with $\mathrm{Si} / \mathrm{Al}=$ 1.2 from UOP Antwerpen; and FAUY zeolite with Si/Al $=2.7$ from ZEOLYST). The zeolite powder was suspended in $500 \mathrm{~mL}$ of an aqueous silver nitrate solution containing the desired weight percentage (0.04 - to $0.5 \mathrm{mM}$, Sigma-Aldrich, $99 \%$ purity), the suspension was agitated overnight in the dark using an end-over-end shaker. The powder was recovered by filtration using a Büchner filter and washed several times with deionised water, then the sample was calcined overnight at $450{ }^{\circ} \mathrm{C}\left(5^{\circ} \mathrm{C} /\right.$ minute $)$ following 2 steps of 
15 minutes each at 100 and $150{ }^{\circ} \mathrm{C}$ to prevent any damage in the zeolite structure. After calcination the samples were cooled and stored in the dark for its further analysis. ${ }^{14}$

\section{Steady-state luminescence characterisation.}

For the emission-excitation characterisation, the calcined $\mathrm{Ag}$ containing zeolite samples were placed in a quartz cuvette (1 $\mathrm{mm}$ path length) and sealed by a Teflon stopper. Emission and excitation spectra were recorded using an Edinburgh Instruments FLS 980 fluorimeter (corrected for wavelength dependence throughput and sensitivity of the detection channel). For every excitation wavelength, the emission was collected starting $30 \mathrm{~nm}$ above the excitation wavelength and ending at $800 \mathrm{~nm}$ using $5 \mathrm{~nm}$ steps. The signal above $410 \mathrm{~nm}$ was measured using a $400 \mathrm{~nm}$ long pass glass filter to avoid interference from second-order excitation peaks, the measured intensities were corrected for the transmittance of the long pass filter. The emission was collected in "front face mode" through the quartz cuvette and sent to a PMT for detection. From the separate emission spectra at varying excitation wavelengths, the two dimensional excitation-emission matrices were constructed, the raw data was corrected for background and noise and interpolated to a resolution of $1 \mathrm{~nm} \times 1$ $\mathrm{nm}$.

\section{Photoluminescence quantum yield measurement.}

Absolute photoluminescence yields were measured using an integrating sphere (Labsphere optical Spectralon integrating sphere, $100 \mathrm{~mm}$ diameter) coupled to a fluorimeter (Edinburgh Instruments FLS 980) through optical fibers. ${ }^{10,47}$ The sphere accessories were made from Teflon (sample holder). Two commercial phosphors ( $\mathrm{BaMgAl}_{10} \mathrm{O}_{19}: \mathrm{Eu}^{2+}$, and $\mathrm{BaMgAl}_{10} \mathrm{O}_{19}: \mathrm{Eu}^{2+}, \mathrm{Mn}^{2+}$ ) with known PLQY were measured and used as references for the calibration of the set-up. ${ }^{48}$ 


\section{Photoelectron spectroscopy in air}

The ionisation potentials of the silver-exchanged zeolites were determined by photoelectron spectroscopy in air (PESA) using a Riken Keiki spectrophotometer (Japan) model AC-2. A substrate of evaporated gold on glass was used with the zeolite powder gently compressed on top to form a flat surface of coverage $>1 \mathrm{~cm}^{2}$. The conditions employed during the measurements were a scanning energy range from 4.3 to $6.2 \mathrm{eV}$ with a measurement interval of $0.05 \mathrm{eV}$, an integration time of $100 \mathrm{~s}$ and UV spot intensity of 1500 $\mathrm{nW}$. The resolution of the instrument is $10 \mathrm{meV}$ and the incident UV spectrum is calibrated against a standard photodiode.

\section{X-ray phototoelectron spectroscopy}

X-ray photoelectron spectroscopy was performed on the silver-exchanged zeolite powders with a Thermo Scientific ${ }^{\mathrm{TM}}$ K-Alpha ${ }^{\mathrm{TM}} \mathrm{X}$-ray Photoelectron Spectrometer (XPS) System. The AlK $\alpha$ source produces $\mathrm{x}$-rays $(h v=1486.7 \mathrm{eV})$ that are focussed to a $200 \times 200 \mu \mathrm{m}$ spot (power density $=66 \mathrm{~W} / \mathrm{m}^{2}$ ). Both $\mathrm{Ag} 3 \mathrm{~d}_{5 / 2}$ spectra and Ag Auger spectra were measured with a pass-energy of $50 \mathrm{eV}$ and step of $0.1 \mathrm{eV}$. An electron flood gun was applied during the XPS measurements to minimize charging effects.

\section{Extended X-ray absorption fine structure}

EXAFS data at the Ag K-edge $(\mathrm{E}=25514 \mathrm{eV})$ of heat-treated Ag-exchanged zeolite-FAUY samples were collected at DUBBLE beamline (BM26A) at The European Synchrotron (ESRF, Grenoble, France), operating under beam conditions of $6 \mathrm{GeV}$, in a $7 / 8+1$ mode, at $160-$ $200 \mathrm{~mA}$. This beamline is equipped with a Si(111) double-crystal monochromator and a vertically focusing Si mirror suppressing higher harmonics. EXAFS data of heat-treated 
FAUX $\left[\mathrm{Ag}_{1}\right]$ was collected at GILDA beamline $(\mathrm{BM08})^{49}$ at The European Synchrotron (ESRF, Grenoble, France) operating in a $24 * 8+1$ mode with a current of $200 \mathrm{~mA}$. The monochromator was equipped with a pair of Si (311) crystals and run in Dynamically Focusing mode. The harmonic rejection was carried out by using a pair of Pt-coated mirrors (Ecut off $=31 \mathrm{keV})$.

EXAFS measurements were performed in ambient conditions on pellets $(1.3 \mathrm{~cm}$ diameter and $1 \mathrm{~mm}$ thickness) which had been prepared with a mechanical press ( 1.5 tons) and stored in the dark prior measurements. Data were collected in transmission mode up to a wave number $k=13 \AA^{-1}$ with typical acquisition times of $45 \mathrm{~min}$ (i.e. 1-25 s per data point). Three spectra were averaged to improve the signal to noise ratio.

Data reduction of the experimental X-ray absorption spectra was performed with the program EXBROOK. Background subtraction and normalization was carried out by fitting (i) a linear polynomial to the pre-edge region in order to remove any instrumental background and absorption effects from other edges and (ii) cubic splines simulating the absorption coefficient from an isolated atom to the post-edge region. EXAFS refinements were performed with the EXCURVE package. Phase shifts and backscattering factors were calculated $a b$ initio using Hedin-Lundqvist potentials.

\section{Electron spin resonance}

ESR spectra were recorded on a Bruker 200D-SRC device in X-ban with a sweep of $6800 \mathrm{~T}$ centred at $3450 \mathrm{~T}$, the zeolite samples were loaded into a high quality quartz tubes that were inserted in the double rectangular $\mathrm{TE}_{104}$ cavity of the ESR spectrometer, which was cooled by liquid nitrogen to a temperature of $120 \mathrm{~K}{ }^{14}$ 
REFERENCES (METHODS)

47. Coutino-Gonzalez E., et al. Thermally activated LTA(Li)-Ag zeolites with water-responsive photoluminescence properties. Journal of Materials Chemistry C 3, 11857-11867 (2015).

48. Leyre S., et al. Absolute determination of photoluminescence quantum efficiency using an integrating sphere setup. Rev. Sci. Instrum. 85, 23115-23115 (2014).

49. d'Acapito F., Trapananti A., Torrengo S. \& Mobilio S. X-ray Absorption Spectroscopy: the Italian beamline GILDA of the ESRF. Notiziario Neutroni e Luce di Sincrotrone 19, 14-23 (2014).

\section{ACKNOWLEDGEMENTS}

This work was financially supported by EC through the projects FP7-NMP-2012 SACS (GA310651), the ERC projects SUPRAFUNCTION (GA-257305), LIGHT (GA-307523) and FLUOROCODE (GA-291593), the Marie-Curie project IEF-MULTITUDES (PIEF-GA-2012326666), the Agence Nationale de la Recherche through the LabEx project Chemistry of Complex Systems (ANR-10-LABX-0026_CSC), the International Center for Frontier Research in Chemistry (icFRC), the 'Fonds voor Wetenschappelijk Onderzoek FWO' (G0990.11, G.0197.11, G.0962.13, G.0B39.15), the Flemish government (long term structural fundingMethusalem grant CASAS METH/08/04), the Flemish 'Strategisch Initiatief Materialen' SoPPoM program, the KU Leuven Research Fund (IDO/07/011), the Hercules foundation (HER/08/21), and the Belgian Federal Science Policy Office (IAP-VI/27). The experiments 2601-865 and CH-4207 were performed on DUBBLE-BM26A and GILDA-BM08 beamlines respectively of The European Synchrotron (ESRF), Grenoble, France. Access to DUBBLE was arranged through the general support of the Fund for Scientific Research-Flanders (FWO) for the use of central facilities. The authors thank the staff of DUBBLE-BM26A and GILDA-BM08 beamlines (ESRF) for their assistance and technical support. We would like to thank UOP 
Antwerpen for their donation of the 3A, 4A, and FAUX zeolites. OF is a Royal Society University Research Fellow.

\section{AUTHOR CONTRIBUTIONS}

PS, MR and JH conceived the experiments. OF, FR and SB conducted the photoelectron spectroscopy experiments. ECG and WB prepared the Ag-zeolites and conducted the optical characterisation. PL, DG and ECG performed the EXAFS measurements and analysis. DDV and ECG performed the ESR measurements and analysis. OF and PS prepared the manuscript with contributions from all co-authors.

a
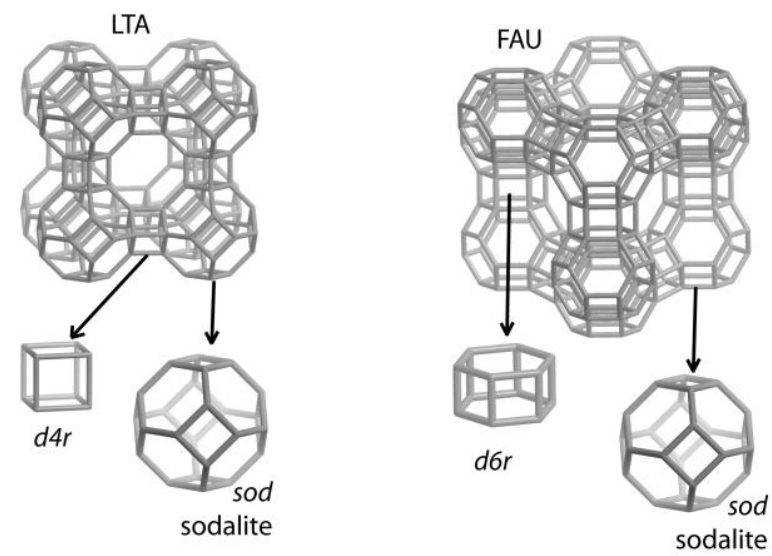

b

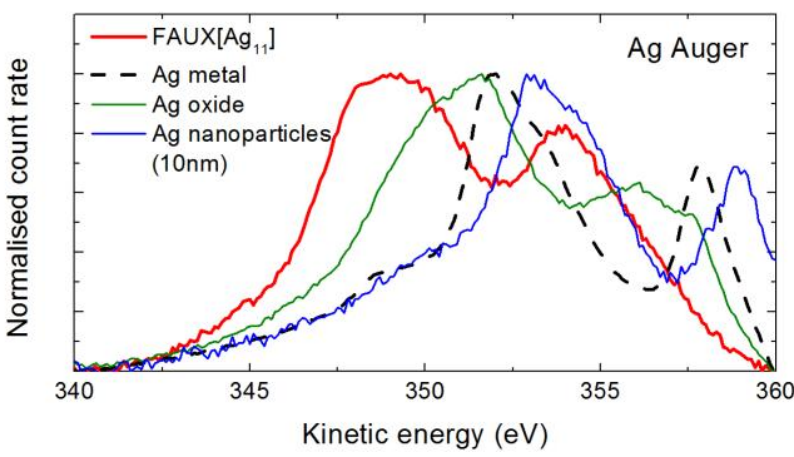

Figure 1 - Topology of the zeolite frameworks and Auger spectra of their silver clusters. a Schematic of the LTA and FAU zeolite frameworks and their building blocks. Vertices of the stick 
model indicate T-atom (Al or Si) positions. For clarity oxygen atoms and the counter-cations are not shown. $\mathbf{b}$ Auger spectra of silver in four forms: metallic, $\mathrm{Ag}(\mathrm{I})$ oxide, $10 \mathrm{~nm}$ sodium citrate stabilised nanoparticles, and as clusters in a calcined zeolite (FAUX[Ag 11$])$. 

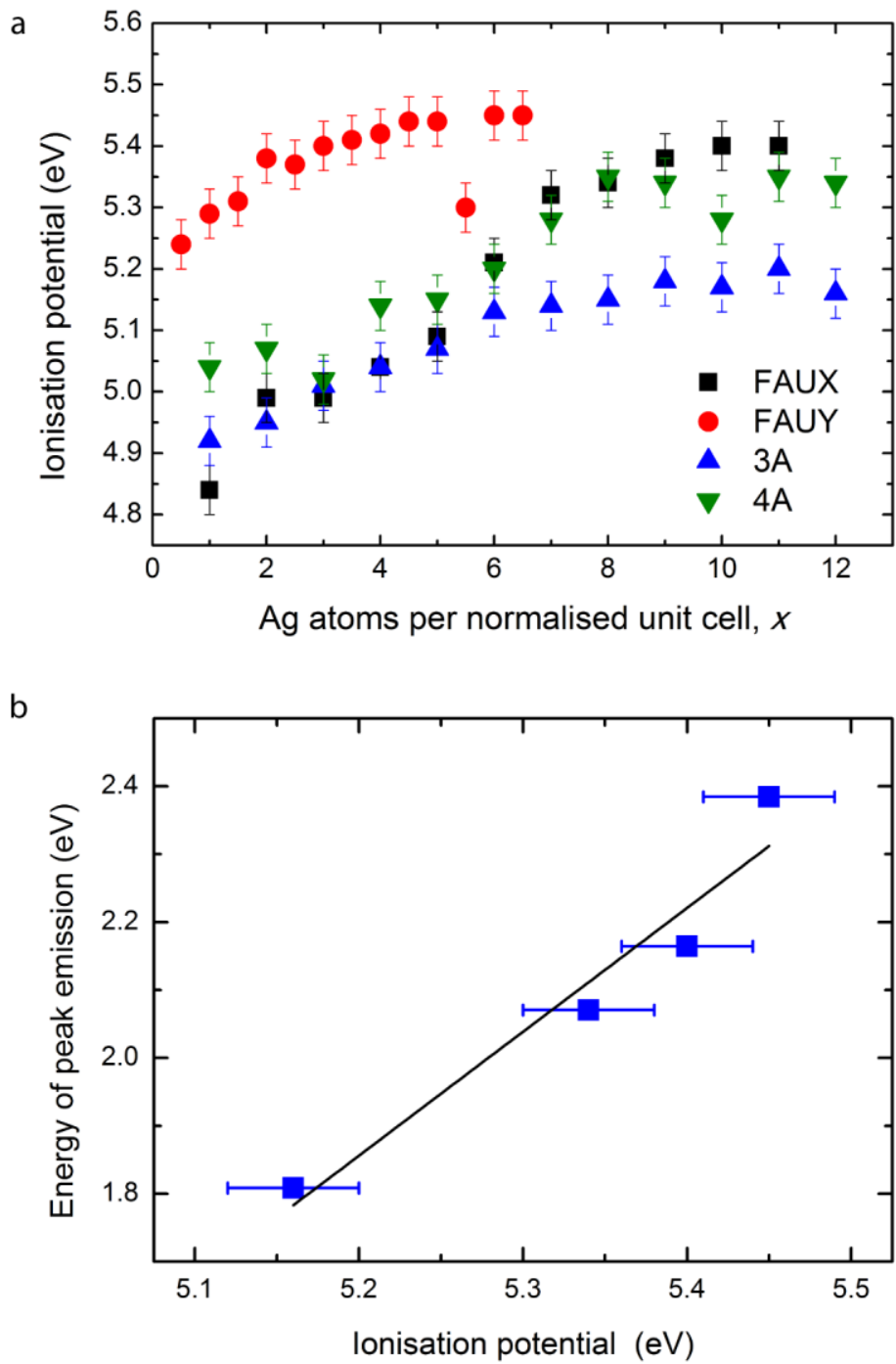

Figure 2 lonisation potentials of the heat-treated silver-exchanged zeolites a lonisation potentials of the four calcined zeolites (FAUX, FAUY, 3A and 4A) across the full range of silver loadings determined from PESA measurements. b Peak emission energy plotted against ionisation potential for the four fully-exchanged zeolites (FAUX[Ag $\left.{ }_{11}\right], F A U Y\left[\mathrm{Ag}_{6.5}\right]$, $3 A\left[\mathrm{Ag}_{12}\right]$ and $\left.4 \mathrm{~A}\left[\mathrm{Ag}_{12}\right]\right)$. The error bars in $\mathbf{a}$ and $\mathbf{b}$ are derived from the scatter between repeated measurements. 

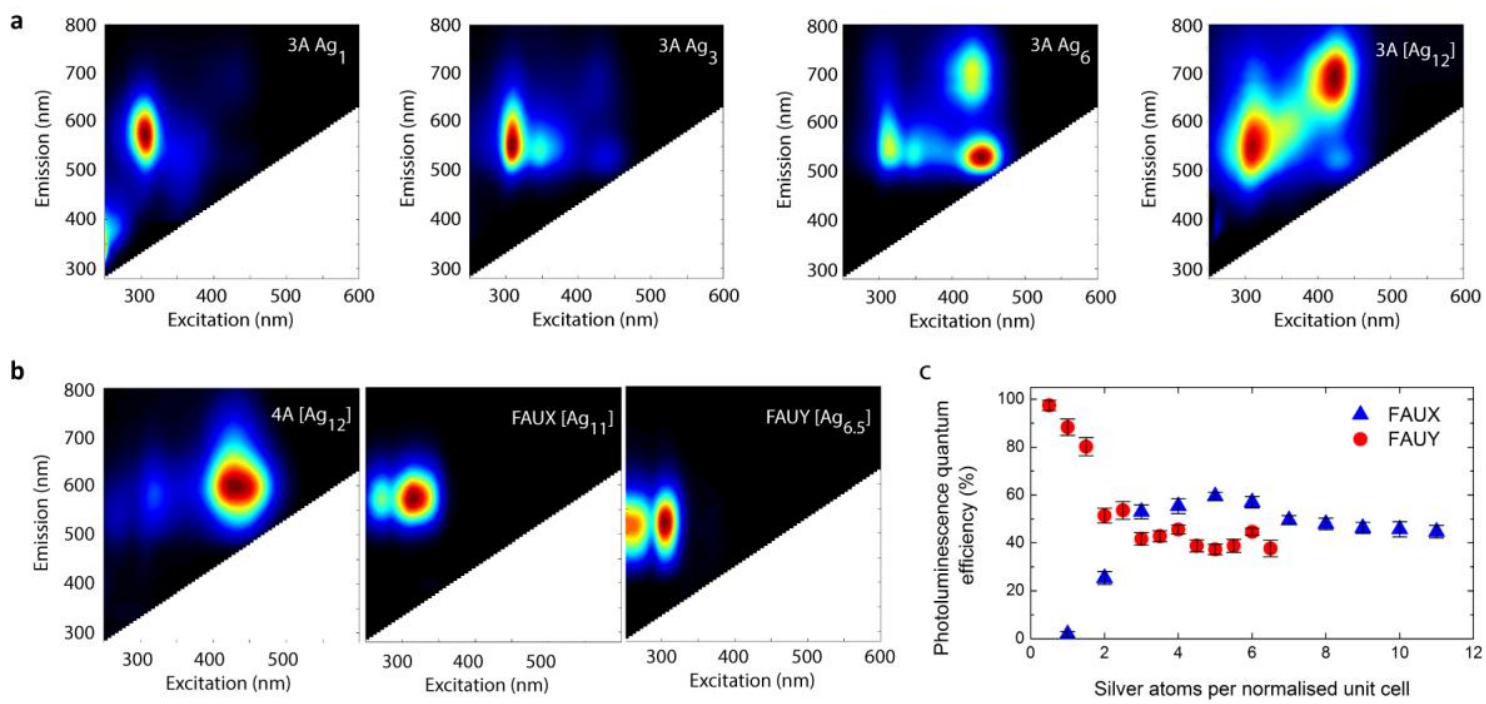

Figure 3 - Photoluminescence properties of heat-treated silver-exchanged zeolites. a Excitation-emission two-dimensional plots of the $3 \mathrm{~A}$ zeolite across silver loadings showing the growth of new peaks with increasing silver loading. $\mathbf{b}$ Excitation-emission twodimensional plots of the fully exchanged and calcined zeolites of 4A, FAUX and FAUY. c Photoluminescence quantum yields $\left(\lambda_{\text {exc }}=305 \mathrm{~nm}\right)$ of the FAU zeolites across the full range of silver loadings showing significant deviation between the FAUX and FAUY zeolites at low silver concentrations. 

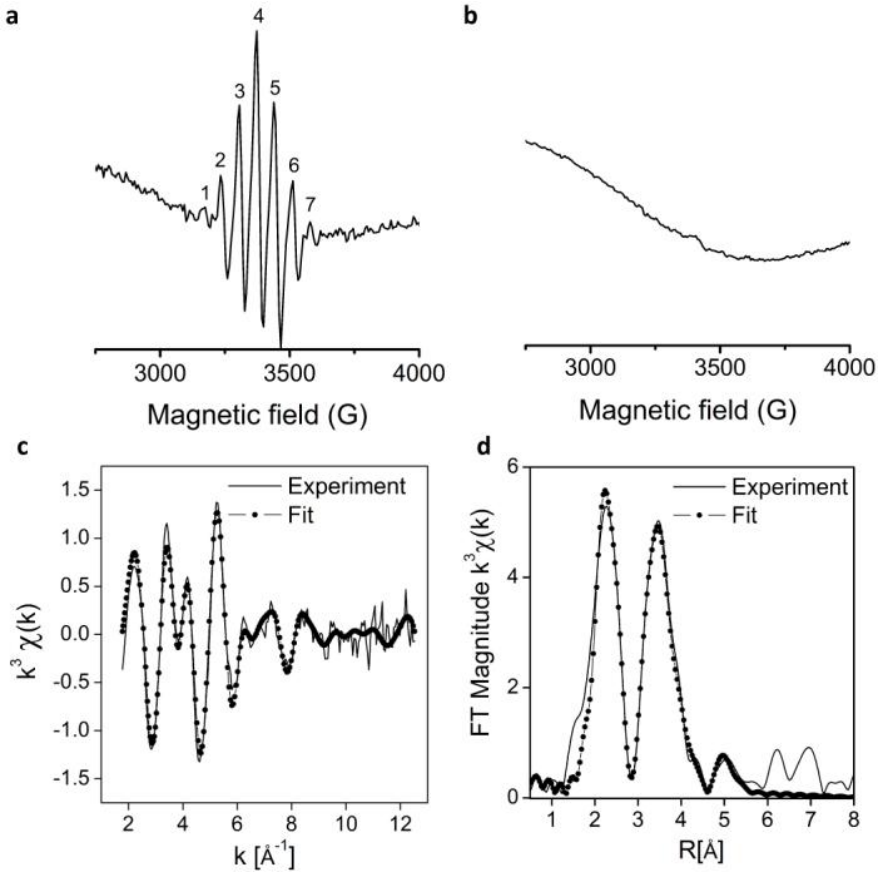

Figure 4 - EXAFS and ESR studies of the silver clusters. ESR of a $3 A\left[\mathrm{Ag}_{6}\right]$ and $\mathbf{b} 3 \mathrm{~A}\left[\mathrm{Ag}_{2}\right]$ showing the presence of a 7-peaked ESR signal in the high silver content $3 A\left[\mathrm{Ag}_{6}\right]$ material and the absence of ESR active silver clusters in the low silver content $3 \mathrm{~A}\left[\mathrm{Ag}_{2}\right]$ material. $\mathrm{k}^{3}$-weighted EXAFS signal $\mathbf{c}$ with the corresponding phase corrected Fourier transform $\mathbf{d}$ of heat-treated FAUY $\left[\mathrm{Ag}_{0.5}\right]$.
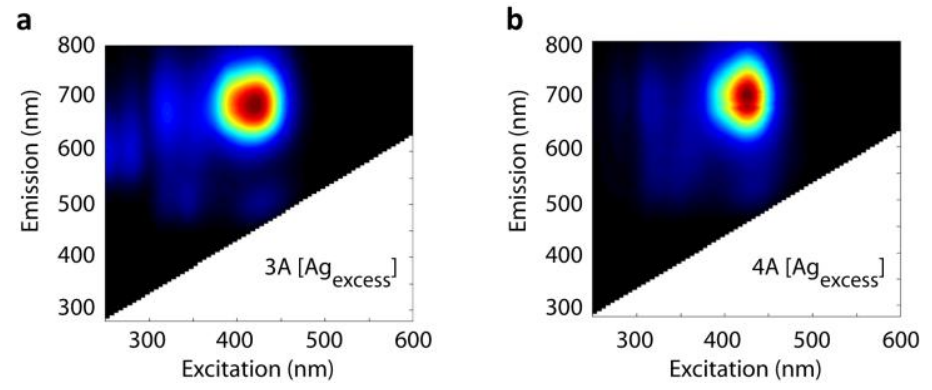

Figure 5-LTA zeolites exchanged in a solution with excess silver ions. Excitation-emission two-dimensional plots of the fully exchanged and calcined zeolites where exchange was performed with an excess concentration of silver nitrate, $\mathbf{a} 3 \mathrm{~A}[\mathrm{Ag}$ excess $]$ and $\mathbf{b} 4 \mathrm{~A}[\mathrm{Ag}$ excess $]$. It can be seen that under these conditions, where all $\mathrm{Na}^{+}$and $\mathrm{K}^{+}$ions have been exchanged, the spectra of the two are similar. 